\title{
On the Minimax Design of Passband Linear-Phase Variable Digital Filters Using Semidefinite Programming
}

\author{
K. M. Tsui, K. S. Yeung, S. C. Chan, and K. W. Tse
}

\begin{abstract}
Variable digital filters (VDFs) are useful to the implementation of digital receivers because its frequency characteristics such as fractional delays and cutoff frequencies can be varied online. In this letter, it is shown that the optimal minimax design of VDFs with passband linear-phase can be formulated and solved as a semi-definite programming (SDP) problem, which is a powerful convex optimization method. In addition, other objective functions, such as least squares, and linear and convex quadratic inequality constraints can readily be incorporated. Design examples using a variable fractional delay (VFD) and a variable cutoff frequency (VCF) FIR filters are given to demonstrate the effectiveness of the proposed approach.
\end{abstract}

Index Terms-Variable digital filter (VDF) design, low-delay, minimax, semidefinite programming (SDP), variable cutoff frequency, variable fractional delay.

\section{INTRODUCTION}

$\mathbf{V}$ ARIABLE digital filters (VDFs) [1] are digital filters with controllable spectral characteristics such as variable cutoff frequency, adjustable passband width [2], [3], controllable fractional delay, etc [4]-[7]. They are useful in arbitrary sample rate changers [8], digital synchronizers [9], and other applications involving online tuning of frequency characteristics.

The least squares design criterion is commonly used to design Farrow-based FIR VDFs [2], [10], [11] because it only involves the solution of a system of linear equation. Linear programming technique [3], [7] has also been proposed to design variable digital filters having linear-phase characteristics and minimax design errors. In this letter, the minimax design of VDFs to achieve a lower system delay in the passband is considered. For convenience, we shall call these filters having approximate linear-phase characteristics in their passband the "passband linear-phase" digital filters. It is shown that the optimal design problem can be formulated as a semidefinite programming (SDP) problem, which is a versatile framework for designing digital FIR and IIR filters [12]-[14]. Alternatively, another efficient convex optimization tool called second-order cone programming (SOCP) [17], which requires shorter design time, can also be used. In SDP, a linear function is minimized subject to the positive semidefinite constraint of a set of symmetric

Manuscript received November 21, 2003; revised January 7, 2004. This work was presented in part at ISCAS'2003 [8]. The associate editor coordinating the review of this paper and approving it for publication was Dr. Xi Zhang.

The authors are with the Department of Electrical and Electronic Engineering, The University of Hong Kong, Hong Kong (e-mail: kmtsui@eee.hku.hk; ksyeung@eee.hku.hk; scchan@eee.hku.hk; kwtse@eee.hku.hk).

Digital Object Identifier 10.1109/LSP.2004.833585 matrices, which are affine functions of the variable. This covers a wide range of practical problems with different design constraints. Moreover, since SDP is a convex optimization problem, if a design problem can be formulated as SDP, it also guarantees the optimality of the solution, if it exists. Interested readers are referred to [15] and [16] for more information of SDP, and its application to the design of FIR and IIR filters [12]-[14]

In the proposed design method, the impulse responses of the VDFs are first expressed as a linear combination of some basis functions and the subfilter coefficients. For simplicity, the polynomial basis function is employed. Using the minimax criterion, the subfilter coefficients are stacked together and solved as a SDP. Two types of VDF, namely passband linearphase digital filters with either variable cutoff frequency or variable fractional delay are considered. Design results show that the proposed method yields VDFs with very good frequency characteristics. Moreover, its flexibility and good performance make it an attractive alternative to traditional least-squares-based design methods. The letter is organized as follows: Section II is devoted to the proposed minimax design of the passband linear-phase FIR VDFs. Design examples are given in Section III. Finally, conclusion is drawn in Section IV.

\section{VDF DESIGN USING SDP}

In a VDF, the desired response $H_{d}(\omega, \phi)$ is a function of a spectral parameter $\phi$ (also known as tuning or control parameters). The spectral characteristics of a VDF can therefore be continuously varied by changing $\phi$. The impulse response of the VDF under consideration is assumed to be a linear combination of some basis functions $\psi_{l}(\phi)$ of the spectral parameters $\phi$ and subfilter coefficients $h_{l}(n)$ [2], and is given by

$$
h(n, \phi)=\sum_{l=0}^{L-1} h_{l}(n) \cdot \psi_{l}(\phi) .
$$

If $h(n, \phi)$ is approximated by a polynomial basis function, the function $\psi_{l}(\phi)$ is simply given by $\phi^{l}$, where $\phi$ is assumed to vary linearly over a finite interval. The z-transform of the polynomial-based VDF is then given by

$$
H(z, \phi)=\sum_{n=0}^{N-1} \sum_{l=0}^{L-1} h_{l}(n) \cdot \phi^{l} z^{-n}=\sum_{l=0}^{L-1} H_{l}(z) \phi^{l}
$$

where $H_{l}(z)=\sum_{n=0}^{N-1} h_{l}(n) \cdot z^{-n}$ are the subfilters. (2.2) suggests an implementation as shown in Fig. 1 called the Farrow 


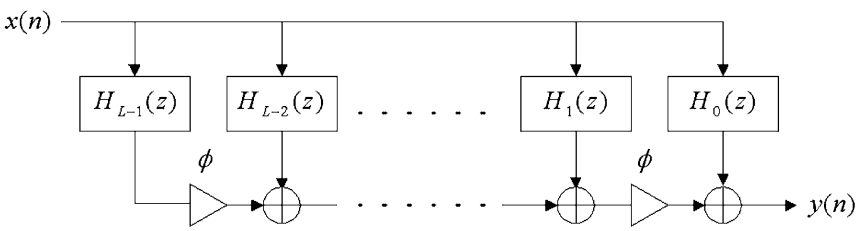

Fig. 1. General Structure of FIR VDF.

structure (please refer to [2] and the references therein for implementing VDFs using the Farrow structure). To simplify the notation, substitute $m=n+N l$ into (2-2), one gets

$$
H\left(e^{j \omega}, \phi\right)=\sum_{m=0}^{L N-1} a_{m} \phi^{l} e^{-j \omega n}
$$

where $n=\bmod (m, N)$ and $l=(m-n) / N$. (2-3) can be rewritten in matrix form as follows:

$$
H\left(e^{j \omega}, \phi\right)=\boldsymbol{a}^{\boldsymbol{T}}\{\mathbf{c}(\omega, \phi)-j s(\omega, \phi)\}
$$

where

$$
\begin{aligned}
\boldsymbol{a} & =\left[a_{0}, \ldots, a_{L N-1}\right]^{T}=\left[\boldsymbol{h}_{\mathbf{0}}^{T}, \ldots, \boldsymbol{h}_{L-1}^{T}\right]^{T} \\
\boldsymbol{h}_{l} & =\left[h_{l}(0), \ldots, h_{l}(N-1)\right]^{T} \text { for } \\
l & =0,1, \ldots, L-1 \\
\boldsymbol{c}(\omega, \phi) & =\left[\boldsymbol{c}_{\boldsymbol{0}}(\omega, \phi)^{T}, \ldots, \boldsymbol{c}_{L-1}(\omega, \phi)^{T}\right]^{T} \\
\boldsymbol{c}_{l}(\omega, \phi) & =\phi^{l}[1, \cos (\omega) \ldots, \cos ((N-1) \omega)]^{T} \text { for } \\
l & =0,1, \ldots, L-1 \\
\boldsymbol{s}(\omega, \phi) & =\left[\boldsymbol{s}_{\mathbf{0}}(\omega, \phi)^{T}, \ldots, \boldsymbol{s}_{L-1}(\omega, \phi)^{T}\right]^{T} \\
\boldsymbol{s}_{l}(\omega, \phi) & =\phi^{l}[0, \sin (\omega) \ldots, \sin ((N-1) \omega)]^{T} \text { for } \\
l & =0,1, \ldots, L-1 .
\end{aligned}
$$

We want to approximate the desired response $H_{d}(z, \phi)$ by $H(z, \phi)$ in the minimax sense. That is

$$
\begin{array}{r}
\min _{\boldsymbol{a}} \max _{(\omega, \phi)}\left\{W(\omega, \phi)\left|H\left(e^{j \omega}, \phi\right)-H_{d}\left(e^{j \omega}, \phi\right)\right|\right\}, \\
(\omega, \phi) \in \Omega
\end{array}
$$

where $W(\omega, \phi)$ is a positive weighting function, and $\Omega$ is the (frequency, tuning range) of interest. Let $H_{R_{-} d}(\omega, \phi)$ and $H_{I-d}(\omega, \phi)$ be the real and imaginary parts of $H_{d}\left(e^{j \omega}, \phi\right)$, the minimization problem in (2-5) can be reformulated as

$$
\begin{array}{r}
\min _{\boldsymbol{a}} \delta \text { subject to } \delta-\left[\alpha_{R}^{2}(\omega, \phi)+\alpha_{I}^{2}(\omega, \phi)\right] \geq 0, \\
(\omega, \phi) \in \Omega
\end{array}
$$

where $\alpha_{R}(\omega, \phi)=W(\omega, \phi) \cdot\left|\boldsymbol{a}^{T} \boldsymbol{c}(\omega, \phi)-H_{R \_d}(\omega, \phi)\right|$ and $\alpha_{I}(\omega, \phi)=W(\omega, \phi) \cdot\left|\boldsymbol{a}^{T} \boldsymbol{s}(\omega, \phi)+H_{I \_d}(\omega, \phi)\right|$.

Using Schur complement [12], the constraints in (2-6) is equivalent to

$$
\begin{aligned}
\boldsymbol{\Gamma}(\boldsymbol{a}, \omega, \phi) & \geq 0 \\
\boldsymbol{\Gamma}(\boldsymbol{a}, \omega, \phi) & =\left[\begin{array}{llc}
\delta & \alpha_{R}(\omega, \phi) & \alpha_{I}(\omega, \phi) \\
\alpha_{R}(\omega, \phi) & 1 & 0 \\
\alpha_{I}(\omega, \phi) & 0 & 1
\end{array}\right] .
\end{aligned}
$$

Digitizing the control parameter $\phi$ and frequency variable $\omega$ over a dense set of frequencies $\left\{\omega_{i}, 1 \leq i \leq K_{1}\right\}$ and $\left\{\phi_{k}, 1 \leq k \leq K_{2}\right\}$ on the range of interests, the constraints in (2-7) become

$$
\begin{aligned}
& \boldsymbol{\Gamma}\left(\boldsymbol{a}, \omega_{i}, \phi_{k}\right) \geq 0, \quad\left\{\omega_{i}, 1 \leq i \leq K_{1}\right\} \quad \text { and } \\
& \left\{\phi_{k}, 1 \leq k \leq K_{2}\right\} .
\end{aligned}
$$

It can be shown that (2-9) can be stacked together to form the following set of linear matrix inequalities:

$$
\begin{aligned}
& \boldsymbol{\Gamma}_{i, k}(\boldsymbol{a}) \geq 0, \quad \text { where } \\
& \boldsymbol{\Gamma}_{i, k}(\boldsymbol{a})=\operatorname{diag}\left\{\boldsymbol{\Gamma}\left(\boldsymbol{a}, \omega_{1}, \phi_{1}\right), \ldots, \boldsymbol{\Gamma}\left(\boldsymbol{a}, \omega_{K_{1}}, \phi_{K_{2}}\right)\right\} .
\end{aligned}
$$

Additional linear and convex quadratic constraints can be stacked into $\boldsymbol{\Gamma}_{i, k}(\boldsymbol{a}) \geq 0$ as in (2-9). By defining the augmented variable $\boldsymbol{x}=\left[\begin{array}{ll}\delta & \boldsymbol{a}^{T}\end{array}\right]^{T},(2-6)$ can be cast to the standard SDP problem as follows:

$$
\underset{\boldsymbol{x}}{\operatorname{minimize}} \quad \boldsymbol{c}^{T} \boldsymbol{x} \quad \text { subject to } \boldsymbol{\Gamma}(\boldsymbol{x}) \geq 0
$$

where $\boldsymbol{c}=[1,0, \ldots 0]^{T}$. Alternatively, the least square stopband attenuation can be minimized

$$
E_{\mathrm{LS}}(\boldsymbol{a})=\int_{\Omega} W(\omega, \phi)\left|H\left(e^{j \omega}, \phi\right)-H_{d}(\omega, \phi)\right|^{2} d \omega d \phi .
$$

This is a quadratic programming problem

$$
\min _{\boldsymbol{a}} \boldsymbol{a}^{T} \boldsymbol{Q} a+2 \boldsymbol{a}^{T} \boldsymbol{g}+k
$$

where

$$
\begin{aligned}
Q= & \int_{\Omega} W(\omega, \phi) \cdot\left[\boldsymbol{c}(\omega, \phi) \boldsymbol{c}(\omega, \phi)^{T}\right. \\
& \left.+\boldsymbol{s}(\omega, \phi) \boldsymbol{s}(\omega, \phi)^{T}\right] d \omega d \phi, \\
\boldsymbol{g}= & \int_{\Omega} W(\omega, \phi)\left[\boldsymbol{c}(\omega, \phi) H_{R_{-} d}(\omega, \phi)\right. \\
& \left.+\boldsymbol{s}(\omega, \phi) H_{I_{-} d}(\omega, \phi)\right] d \omega d \phi, \text { and } \\
k= & \int_{\Omega} W(\omega, \phi)\left|H_{d}(\omega, \phi)\right|^{2} d \omega d \phi .
\end{aligned}
$$

To formulate (2-12) as a SDP problem, we decompose $Q$ as $Q=\boldsymbol{P}^{T} \boldsymbol{P}$ and reformulate it by means of Schur complement as follows:

$$
\min _{\boldsymbol{x}} \boldsymbol{c}^{T} \boldsymbol{x} \quad \text { subject to }\left[\begin{array}{lc}
\delta+2 \boldsymbol{a}^{T} \boldsymbol{g}-k & \boldsymbol{a}^{T} \boldsymbol{P}^{T} \\
\boldsymbol{P a} & 1
\end{array}\right] \geq 0 .
$$

By employing different desired frequency response $H_{d}\left(e^{j \omega}, \phi\right)$, digital filters with various tunable spectral characteristics can be designed. In addition, linear and convex quadratic constraints can be stacked with (2-13) and solved using SDP. As an illustration, let us consider the design of passband linear-phase lowpass FIR filters with either variable cutoff frequency or variable fractional delay. The desired response of a variable cutoff frequency digital filter (VCF-DF) can be written as

$$
H_{d}\left(e^{j \omega}, \phi\right)= \begin{cases}e^{-j \omega \tau}, & 0<|\omega|<\omega_{p}(\phi) \\ 0, & \omega_{s}(\phi)<|\omega|<\pi\end{cases}
$$


where $\tau=(N-1) / 2-D$ is the group delay; $D$ is the delay reduction parameter compared with its linear-phase counterpart $(D=0$ corresponds to a linear-phase VCF-DF $) ; \omega_{p}(\phi)=\left(\omega_{p}+\right.$ $\left.\lambda_{p} \phi\right)$ with $\lambda_{p}$ the tuning range in the passband and $\omega_{s}(\phi)=$ $\left(\omega_{s}+\lambda_{s} \phi\right)$ with $\lambda_{s}$ the tuning range in the stopband. Similarly, the desired response of a variable fractional delay digital filter (VFD-DF) is

$$
H_{d}\left(e^{j \omega}, \phi\right)= \begin{cases}e^{-j \omega \tau(\phi)}, & 0<|\omega|<\omega_{p} \\ 0, & \omega_{s}<|\omega|<\pi\end{cases}
$$

where $\tau(\phi)=(N-1) / 2-D+\phi$ is the tunable group delay of the VFD-DF, and $\omega_{p}$ and $\omega_{s}$ are respectively the passband and stopband cutoff frequencies.

\section{DESIGN EXAMPLES}

\section{A. Variable Cutoff Frequency Digital Filter}

In this example, VCF-DFs with minimax design error are designed using the proposed SDP method. The transition bandwidth is fixed at $0.2 \pi$ and the passband edge is varied from $0.2 \pi$ to $0.4 \pi$. There are 50 samples for the tuning parameter $\phi$ within the interval $[0,1]$, and 100 samples for the frequency of interest. The VCF-DFs have a subfilter length of 31 and an interpolation order of 5 for the polynomial basis function. Therefore, the passband group delay of the linear-phase VCF-DF is 15 with the delay reduction parameter $D=0$. Fig. 2(a) shows the frequency response of the linear-phase VCF-DF so obtained. The corresponding passband deviation and stopband attenuation are 0.0293 and $49.3326 \mathrm{~dB}$, respectively. As a comparison, a low-delay VCF-DF is also designed with $D=3$. Hence, the overall system delay is reduced to 12 . The frequency and the group delay responses of the low-delay VCF-DF so obtained are plotted in Fig. 2(b) and (c) respectively. The corresponding passband deviation and stopband attenuation are 0.0303 and $48.9375 \mathrm{~dB}$, which are slightly worse than those of its linearphase counterpart. The worst-case passband group delay error is 0.1746 samples. The design time for this example is about 14.7 min on a Pentium 4 computer. For comparison, we have also implemented the method in [3] for the linear-phase case. Our result is similar to that obtained by the linear programming (LP) approach proposed in [3]. This is to be expected because SDP is a generalization of linear programming. Due to page limitation, details are omitted. On the other hand, unlike SDP, LP is only applicable to the design of linear-phase VCF-DF, but not passband linear-phase VDFs considered in this paper. It should be noted that there is a tradeoff between passband delays and performance in designing VDFs. For example, when the delay parameter $D$ above is increased to 6 , the corresponding passband deviation, stopband attenuation and group delay error will become $0.044,45.8832$, and 0.2676 samples, respectively. The passband delay, on the other hand, will be lowered to approximately nine samples.

\section{B. Variable Fractional Delay Digital Filter}

In this example, both linear-phase and low-delay VFD-DFs with minimax design error are designed. The passband width under consideration is from 0 to $0.8 \pi$, and it is digitized into 100 evenly spaced sample points. The tuning parameter $\phi$ is in

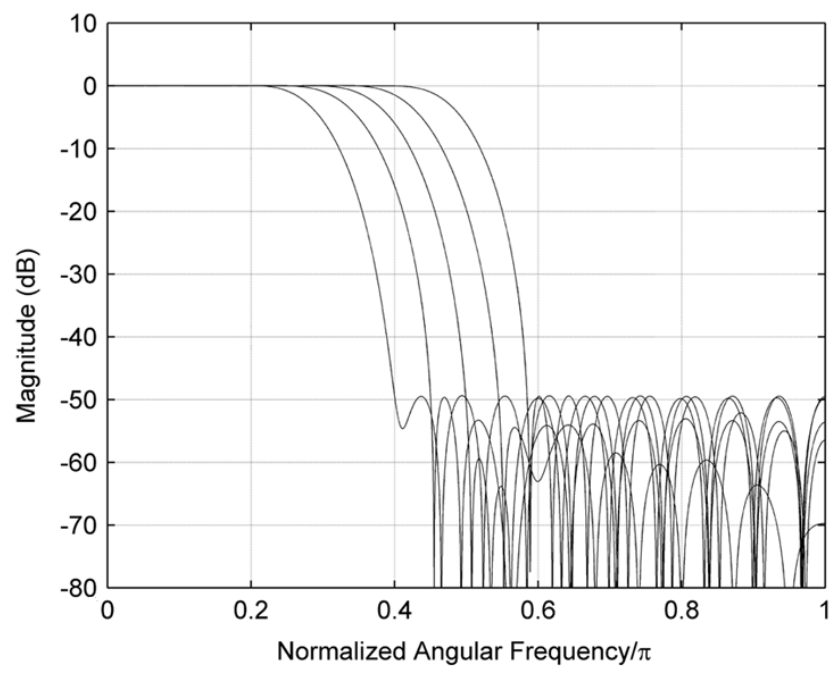

(a)

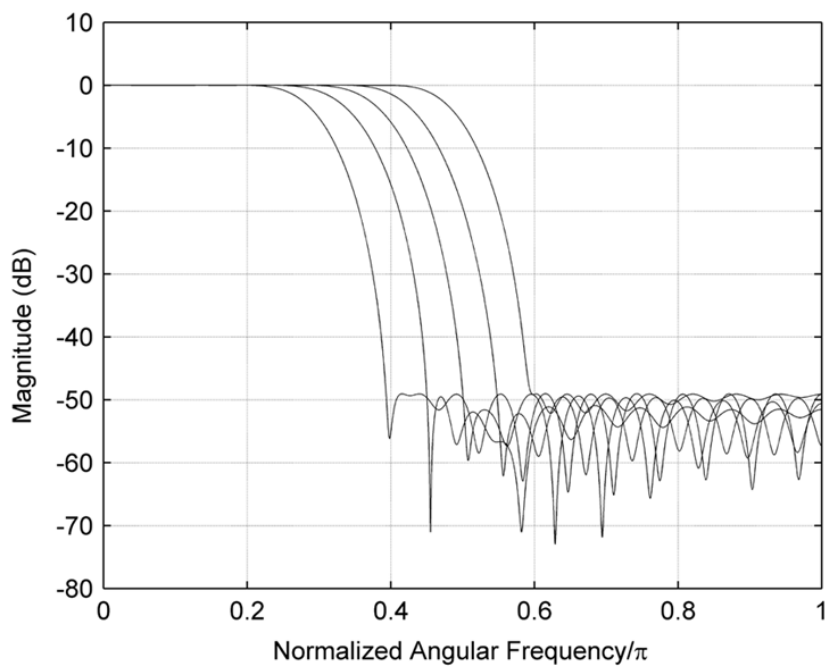

(b)

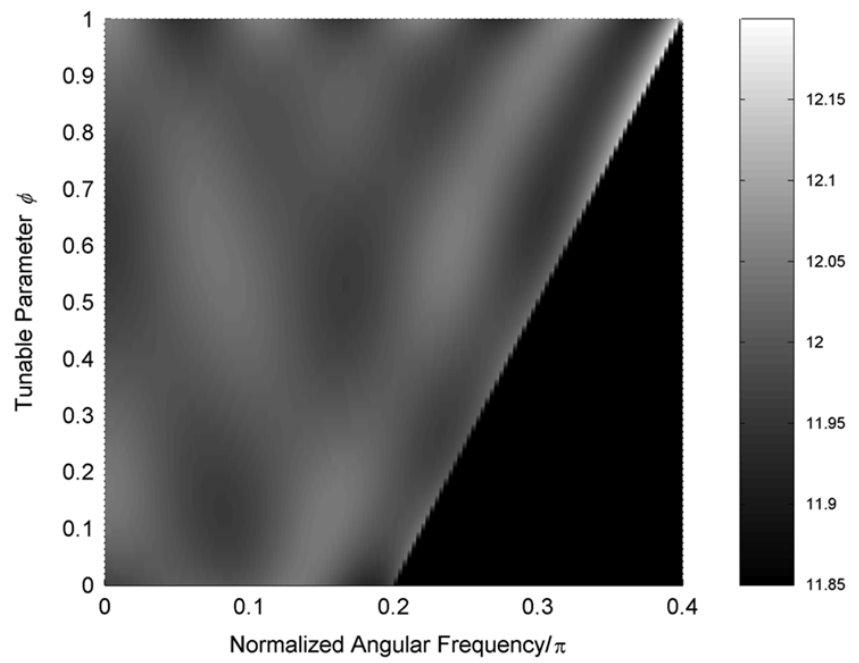

(c)

Fig. 2. Design results in example A. (a) Frequency response of linear-phase variable cutoff frequency digital filter. (b) and (c) Frequency and group delay responses of low-delay variable cutoff frequency digital filter (delay reduction parameter $D=3$ ).

the interval $[-0.5,0.5]$, and it is digitized into 50 evenly spaced sample points. The length of subfilter and the interpolation order 

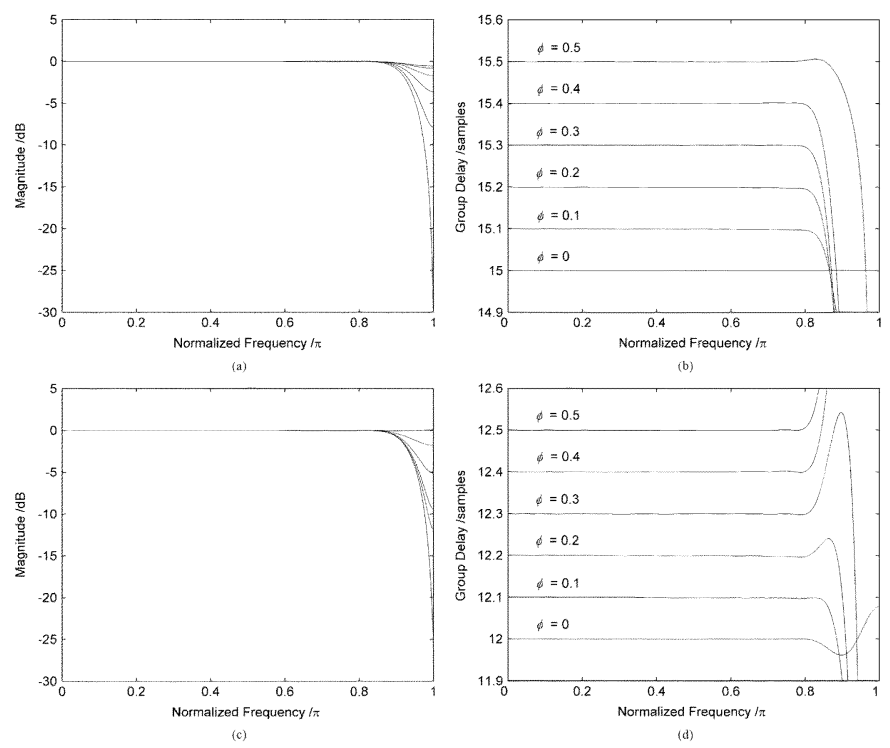

Fig. 3. Design results in example B. (a) and (b) Frequency and group delay responses of linear-phase variable fractional delay digital filter. (c) and (d) Frequency and group delay responses of low-delay variable fractional delay digital filter (delay reduction parameter $D=3$ ).

are 31 and 5, respectively. The delay reduction parameter $D$ of the low-delay VFD-DF is chosen as 3 so that its passband group delay is 12 . The frequency and group delay responses of the linear-phase (low-delay) VFD-DF with $\phi=0,0.1,0.2,0.3,0.4$, and 0.5 are shown in Fig. 3(a), (c) and (b), (d), respectively. For the linear-phase VFD-DF, the corresponding passband deviation and group delay error are 0.0125 - and 0.0032-dB samples, respectively, while for the low-delay VFD-DF, they are found to be $0.01257-$ and $0.0036-\mathrm{dB}$ samples, respectively. The design time for this example is about $14.7 \mathrm{~min}$. Again, it is noticed that the system delay can be reduced at the expense of slightly degradation of filter performance.

\section{CONCLUSION}

A new VDF design approach using SDP is presented. The proposed SDP formulation allows us to design the optimal passband linear-phase VDFs in the minimax and least square sense. Moreover, additional linear and convex quadratic inequality constraints can readily be incorporated. Two common types of VDFs, namely VCF-DFs and VDF-DFs, are designed to illustrate the effectiveness of the proposed approach. Design results show that the SDP method offers an attractive alternative to traditional design methods because of its optimality, generality, and flexibility.

\section{REFERENCES}

[1] R. Zarour and M. M. Fahmy, "A design technique for variable digital filters," IEEE Trans. Circuits Syst. II, vol. 36, pp. 1473-1478, Nov. 1989.

[2] K. S. Pun, S. C. Chan, K. S. Yeung, and K. L. Ho, "On the design and implementation of FIR and IIR digital filters with variable frequency characteristics," IEEE Trans. Circuits Syst. II, vol. 49, pp. 689-703, Nov. 2002.

[3] P. Lowenborg and H. Johansson, "Linear programming design of linearphase FIR filters with variable bandwidth," in Proc. ISCAS'2003, vol. 3, May 2003, pp. 554-557.

[4] C. W. Farrow, "A continuously variable digital delay element," in Proc. ISCAS, vol. 3, 1988, pp. 2641-2645.

[5] J. Vesma and T. Saramaki, "Design and properties of polynomial-based fractional delay filters," in Proc. ISCAS 2000, pp. 104-107.

[6] T. I. Laakso, V. Valimaki, M. Karjalainen, and U. K. Laine, "Splitting the unit delay, tools for fractional delay filter design," IEEE Signal Processing Mag., pp. 30-60, Jan. 1996.

[7] H. Johansson and P. Lowenborg, "On the design of adjustable fractional delay FIR filters," IEEE Trans. Circuits Syst. II, vol. 50, pp. 164-169, Apr. 2003.

[8] S. C. Chan and K. S. Yeung, "On the application of variable digital filters (VDF) to the realization of software radio receivers," in Proc. ISCAS'2003, vol. 3, May 2003, pp. 562-565.

[9] L. Erup, F. M. Gardner, and R. A. Harris, "Interpolation in digital modems. II. Implementation and performance," IEEE Trans. Commun., vol. 41, pp. 998-1008, June 1993.

[10] A. Tarczynski, G. D. Cain, E. Hermanowicz, and M. Rojewski, "WLS design of variable frequency response FIR filters," in Proc. ISCAS 1997, vol. 4, 1997, pp. 2244-2247.

[11] W. S. Lu and T. B. Deng, "An improved weighted least-squares design for variable fractional delay FIR filters," IEEE Trans. Circuits Syst. II, vol. 9, pp. 689-685, Sept. 1997.

[12] W. S. Lu, "Design of nonlinear-phase FIR digital filters: A semidefinite programming approach," in Proc. ISCAS'99, vol. III, Orlando, FL, May 1999, pp. 263-266.

[13] S. P. Wu, S. Boyd, and L. Vandenberghe, "FIR filter design via semidefinite programming and spectral factorization," in Proc. 5th Conf. Decision and Control, Kobe, Japan, Dec. 1996, pp. 271-276.

[14] W. S. Lu and A. Antoniou, "Design of digital filters and filter banks by optimization: A state of the art review," in Proc. EUSIPCO'2000, vol. 1, Tampere, Finland, Sept. 2000, pp. 351-354.

[15] L. Vandenberghe and S. Boyd, "Semidefinite programming," SIAM Rev., vol. 38, pp. 49-95, Mar. 1996.

[16] Handbook of Semidefinite Programming: Theory, Algorithms, and Applications, Kluwer, Norwell, MA, 2000. H. Wolkowicz, R. Saigal, and L. Vandenberge.

[17] K. M. Tsui, S. C. Chan, and K. W. Tse, "Design of complex-valued variable digital filters and its application to the realization of arbitrary sampling rate conversion for complex signals," in Proc. MWSCAS'2004, vol. 2, July 2004 , pp. $349-352$. 